\title{
NUEVOS DATOS SOBRE LOS ISÓPODOS \\ (CRUSTACEA: PERACARIDA) EN LAS COSTAS DEL NOROESTE DE LA PENÍNSULA IBÉRICA
}

\author{
P. Reboreda (*) y V. Urgorri (*)
}

\begin{abstract}
RESUMEN
En el presente trabajo relacionamos un grupo de 11 especies, pertenecientes a cinco géneros, de las cuales cinco, Cymodoce robusta, Janiropsis breviremis, Janira alta, Paramunna bilobata y Eurydice affinis, eran desconocidas en la Península Ibérica; cuatro, Cymodoce emarginata, Cymodoce tattersalli, Cymodoce tuberculata y Jaera hopeana, no habian sido mencionadas en el Atlántico ibérico y una, Ischyromene lacazei, supone la primera cita en aguas gallegas. Se amplían los límites de distribución meridional de cuatro especies, Janiropsis breviremis, Janira alta, Jaera nordica nordica y Paramunna bilobata, y el septemtrional de una, Cymodoce robusta. Asimismo se aportan aspectos relativos a la anatomía y biología de las especies.

Palabras clave: Isopoda, Peracarida, Península Ibérica, distribución
\end{abstract}

\section{ABSTRACT \\ New data about isopods (Crustacea: Peracarida) at the northwest of the Iberian Peninsula coast}

In the present work, we list a group of eleven species, wich belong to five genera. Five of these species, Cymodoce robusta, Janiropsis breviremis, Janira alta, Paramunna bilobata and Eurydice affinis, were unknown in the Iberian Peninsula, four species, Cymodoce emarginata, Cymodoce tattersalli, Cymodoce tuberculata and Jaera hopeana, have never been mentioned at the Iberian Atlantic coast, and one species, Ischyromene lacazei, is recorded for the first time in the Galician waters. The southern limit of four species, Janiropsis breviremis, Janira alta, Jaera nordica nordica and Paramunna bilobata, is extended and the northern limit of distribution of Cymodoce robusta is extended. Besides, we add data about anatomy and biology of the species.

Key words: Isopoda, Peracarida, Iberian Peninsula, distribution

\section{Introducción}

La mayoría de las menciones del Orden Isopoda en la Península Ibérica están inmersas en trabajos generales sobre comunidades bentónicas intermareales, dinámica de poblaciones y estructura trófi- ca, o bien en recopilaciones de material depositado en museos. No obstante, Castelló (1984, 1985, 1986a, b) estudia los Isópodos de la costa catalana y del archipiélago balear, constituyendo las más importantes aportaciones sobre este orden en la Península Ibérica. Posteriormente, Junoy \& Vieitez

\footnotetext{
* Departamento de Bioloxía Animal, Faculdade de Bioloxía, Universidade de Santiago, Campus Sur. 15706 Santiago de Compostela
} 


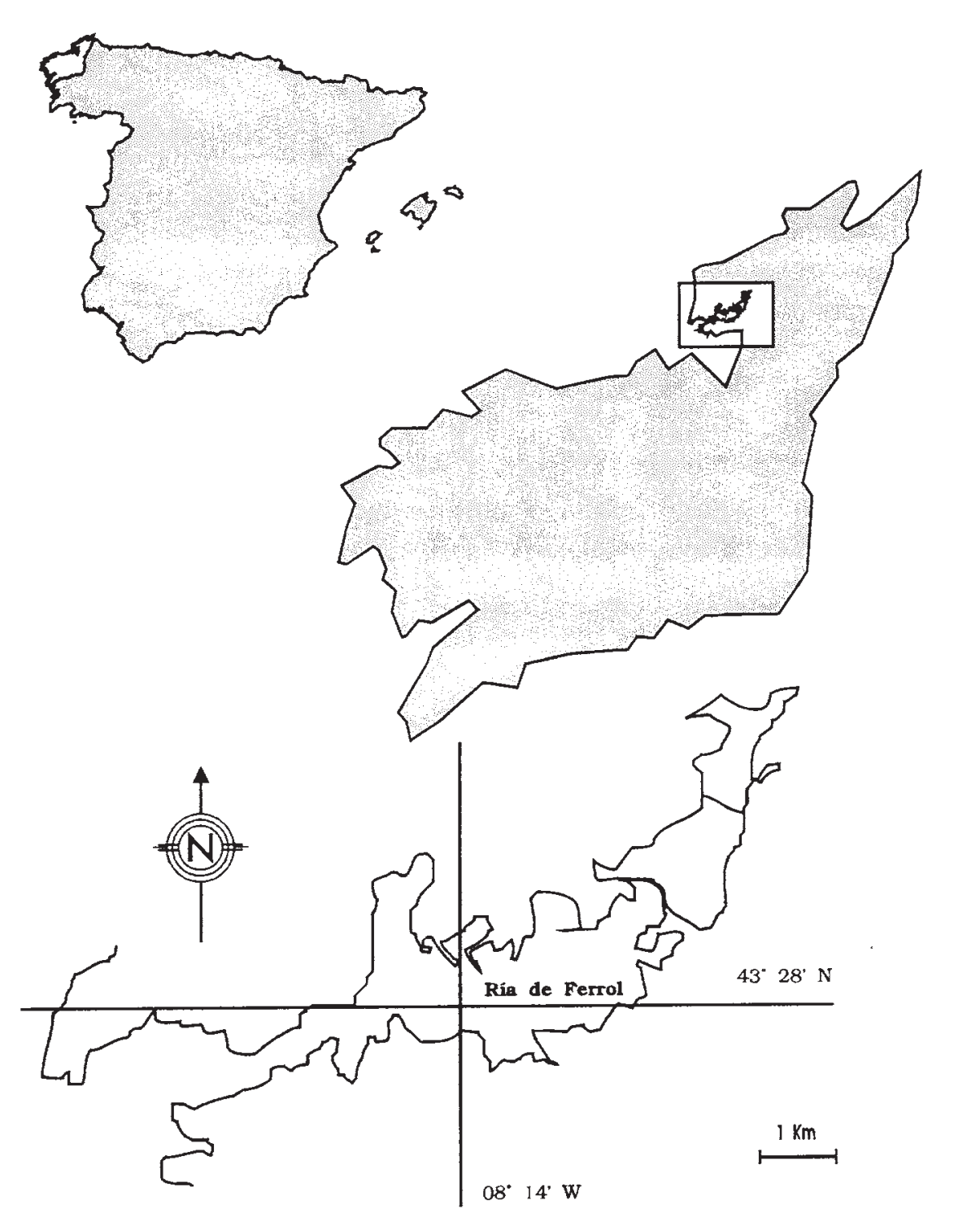

Fig. 1.- Localización geográfica de la Ría de Ferrol.

Fig. 1.- Geographic location of Ferrol estuary (firth).

$(1988,1990)$ realizan estudios sobre los Isópodos en la Ría de Foz (Galicia) e Iborra \& Ros (1984) y Ros et al. (1987) sobre la fauna isopodológica del mar Menor. El trabajo más reciente se debe a Franch \& Ballesteros (1993) sobre los Isópodos de Formentera.

Ante la escasez de datos, sobre todo en la costa atlántica ibérica, abordamos el estudio del Orden
Isopoda en la Ría de Ferrol (Galicia) (Fig. 1), eligiéndola por presentar una configuración recortada que provoca un régimen peculiar de corrientes, que le confieren una gran variedad de fondos y unas características muy variadas y particulares en cuanto al grado de exposición al oleaje y salinidad. Por ello, durante los años 1985-1990, se realizaron numerosos muestreos, intermareales e infralitora- 


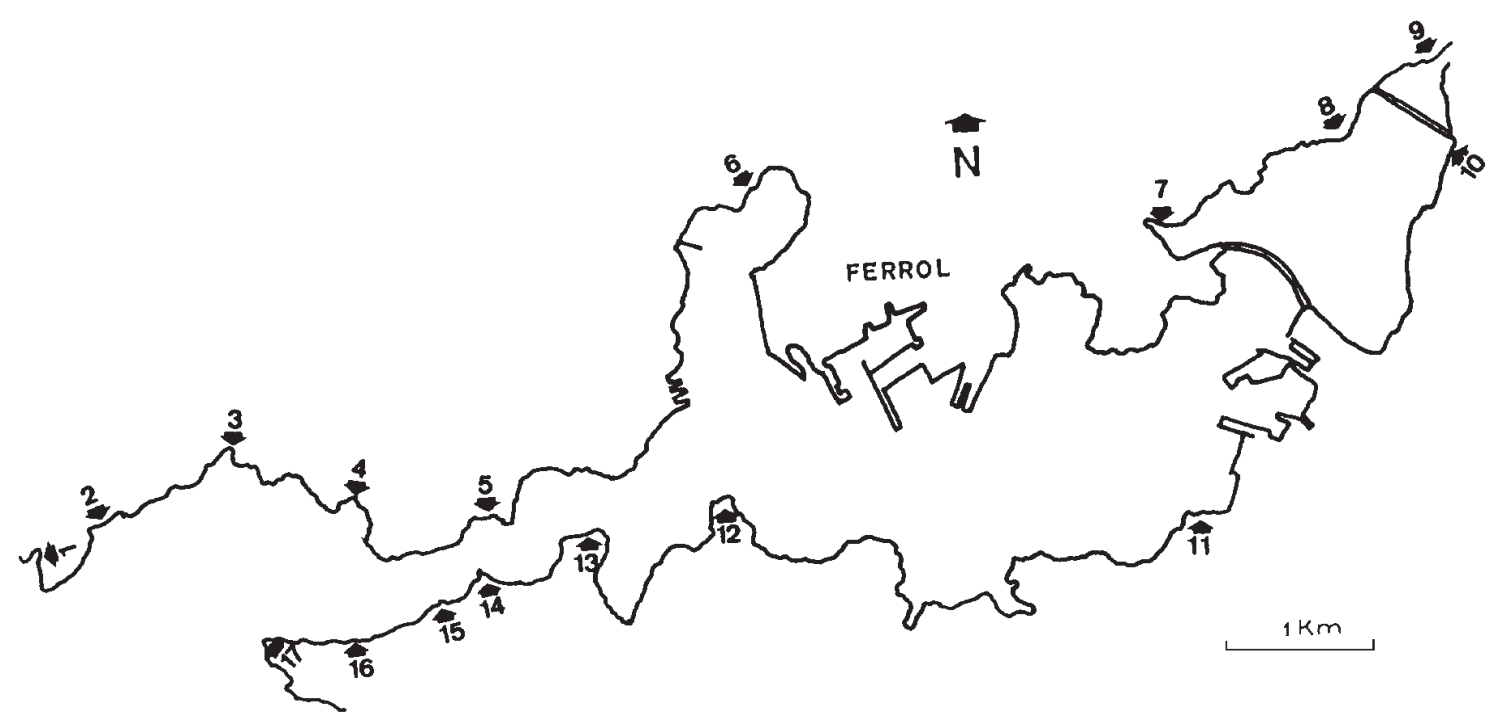

Fig. 2.- Localización de todas las estaciones intermareales donde se efectuaron los muestreos. (01) Prioriño. (02) Canelas, (03) Cariño, (04) Fornelos, (05) Laxe, (06) A Cabana, (07) A Gándara, (08) A Faisca, (09) Xubias, (10) Neda, (11) Maniños, (12) Mugardos, (13) A Redonda, (14) Batel, (15) San Martín, (16) Cabaliños y (17) O Segaño.

Fig. 2.- Location of intertidal positions where samples were taken.

les, encaminados a conocer su fauna isopodológica, obteniéndose un total de 39 especies, de las que varias resultaron ser nuevas menciones para nuestras costas.

\section{Material y Métodos}

A) RECOGIDA DE MUESTRAS INTERMAREALES.- Las muestras proceden de un total de 17 estaciones (Fig. 2; Apéndice) situadas a lo largo de ambos márgenes de la ría y que representan las distintas condiciones ecológicas que en ella se dan. En cada estación se establecieron los distintos niveles intermareales determinados por las cinturas de vegetación, siguiendo el criterio de Wommersley \& Edwards (1952), posteriormente adaptado para aguas ibéricas por Seoane-Camba (1969), PérezCirera \& Maldonado (1982) y Pérez-Cirera \& Pacheco (1985). Debido al tamaño y hábitos de vida de los Isópodos, consideramos insuficiente una recogida de visu y, por lo tanto, se tomó una muestra de cada posible sustrato preferencial (distintos tipos de algas, mejillón, balánidos, esponjas...) al tiempo que se recogían aquellos animales más conspicuos, detectados en un examen minucioso de diferentes sustratos, anotando el nivel intermareal en que se encontraba y las observaciones ecológicas que se creyeron oportunas (inclinación, exposición a la luz u oleaje, sedimento circundante...).

B) ReCogida De muestras infralitorales. - Se dividió la ría en cuadrículas de $1 / 3 \times 1 / 2$ de minuto de lado (Fig. 3; Apéndice), obteniéndose un total de 78 localidades infralitorales (de las cuales tres: 39 , 40 y 43 , no pudieron ser estudiadas por estar situadas en zona militar) realizándose, en cada una de ellas, una toma de muestras mediante una draga de arrastre bentónica tipo "rectangular de naturalista", obteniéndose un volumen aproximado de 25 litros. El sedimento así obtenido era tamizado, haciéndolo pasar, con agua de mar, por una columna de tamices de 5,2 y $0,5 \mathrm{~mm}$ de malla. 
C) Separación, FiJACión Y CONSERVACión.- Tras el aislamiento de la fauna bajo la lupa binocular, los ejemplares fueron fijados con formaldehído al $4 \%$ neutralizado con tetraborato sódico y posteriormente conservados en alcohol al 70\%, al que se le añadía una pequeña cantidad de glicerina con el fin de mantener la elasticidad de las membranas articulares.

D) Estudio De Los EJeMPlaRes. - Para la determinación específica, se realizó un primer estudio de las características generales externas de los individuos (bajo la lupa binocular o microscopio, según las tallas) y posteriormente se pocedía al aislamiento y estudio microscópico de los diferentes apéndices. Para cada especie se separaron los ejemplares en los diferentes estadios de desarrollo (manca, postmanca, adulto indiferenciado, premacho, macho, hembra grávida, hembra en reposo y hembra postgrávida) anotando el número de individuos por estadio y muestra, así como la talla máxima y mínima, tanto en longitud como en anchura.

\section{Resultados}

LISTA DE ESPECIES ENCONTRADAS EN LA RÍA DE FERROL. - Se marcan con * aquellas que constituyen hallazgos faunísticos de interés y que serán objeto de comentarios a continuación.

Orden ISOPODA Latreille, 1817

Suborden GNATHIIDEA Leach, 1814

Familia GNATHIIDAE Harger, 1880

Género Gnathia sensu stricto Monod, 1926

Gnathia maxilaris (Montagu, 1804)

Género Paragnathia Omer-Cooper, 1916

Paragnathia formica (Hesse, 1864)

Suborden ANTHURIDEA Leach, 1814

Familia ANTHURIDAE Leach, 1814

Género Anthura Leach, 1814

Anthura gracilis (Montagu, 1808)

Género Amakusanthura Nunomura, 1977

Amakusanthura iberica Reboreda \& Wagele, 1992

Género Cyathura Norman \& Stebbing, 1886

Cyathura carinata (Kröyer, 1874)
Familia PARANTHURIDAE Menzies \& Glinn, 1968

Género Paranthura Bate \& Westwood, 1868

Paranthura costana Bate \& Westwood, 1868

Paranthura nigropunctata (Lucas, 1849)

Suborden FLABELLIFERA Sars, 1882

Familia CIROLAIDAE Dana, 1853

Género Conilera Leach, 1818

Conilera cylindracea (Montagu, 1804)

Género Eurydice Leach, 1815

*Euridice affinis Hansen 1905

Familia SPHAEROMATIDAE (Dahl, 1916)

Subfamilia CASSIDININAE Iverson, 1982

Género Anoplocopea Racovitza, 1908

Anoplocopea sp.

Género Campecopea (Montagu, 1804)

Campecopea hirsuta (Montagu, 1804)

Subfamilia DYNAMENINAE Bowman, 1981

Género Dynamene Leach, 1814

Dynamene bidentata (Adams, 1800)

Dynamene magnitorata Holdich, 1968

Género Ischyromene Racovitza, 1908

*Ischyromene lacazei Racovitza, 1908

Subfamilia SPHAEROMATINAE Latreille, 1825

Género Cymodoce Leach, 1814

*Cymodoce emarginata sensu Torelli, 1928

*Cymodoce robusta Niestrasz, 1918

*Cymodoce tattersalli Torelli, 1928

Cymodoce truncata Leach, 1814

*Cymodoce tuberculata Costa, 1851

Género Lekanesphaera Verhoeff, 1943

Lekanesphera hookeri hookeri (Leach, 1814)

Lekanesphaera levii (Argano \& Ponticelli, 1981)

Lekanesphera rugicauda (Leach, 1814)

Género Sphaeroma Bosc, 1802

Sphaeroma serratum (Fabricius, 1787)

Suborden ASELLOTA Latreille, 1803

Superfamilia JANIROIDEA Sars, 1899

Familia JANIRIDAE Sars, 1899

Género Jaera Leach, 1813

*Jaera hopeana Costa, 1853

"Grupo albifrons"

Jaera albifrons Leach, 1814

Jaera praehirsuta Forsman, 1949

Jaera posthirsuta Forsman, 1919

"Grupo Nordmanni" 


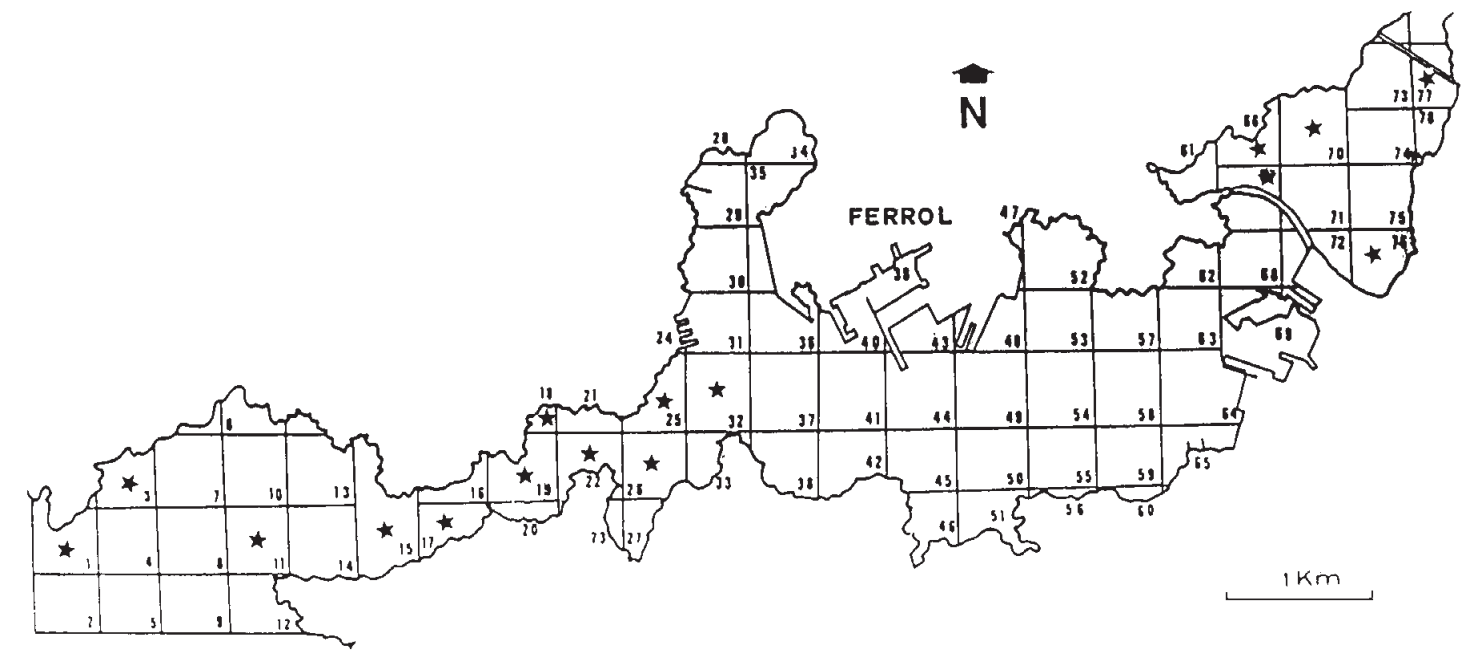

Fig. 3.- Localización de las cuadrículas dragadas. Se señalan con un * aquellas en las que aparecieron Isópodos.

Fig. 3. - Location of dregded areas. Those ones where isopods were found area marked by asterisc (*).

*Jaera nordica nordica Lemercier, 1960

Género Janira Leach, 1814

* Janira alta (Stimpson, 1853)

Janira maculosa Leach, 1814

Género Janiropsis Sars, 1899

*Janiropsis breviremis (Sars, 1882)

Familia MUNNIDAE Sars, 1899

Género Uromunna Menzies, 1962

Uromunna sp.

Familia PARAMUNNIDAE Vanhöffen, 1914

Género Paramunna Sars, 1866

*Paramunna bilobata Sars, 1866

Suborden VALVIFERA Sars, 1882

Familia ARCTURIDAE Sars, 1899

Género Arturella Sars, 1897

Arturella carlosoteroi Reboreda et al. 1994

Familia IDOTEIDAE Milne Edwards, 1840

Género Idotea Fabricius, 1798

Idotea baltica (Pallas, 1772)

Idotea chelipes (Pallas, 1776)

Idotea granulosa Rathke, 1843

Idotea emarginata (Fabricius, 1789)

Idotea pelagica Leach, 1815

Género Synisoma Collinge, 1917

Synisoma acuminata (Leach, 1815)

Synisoma lancifer (Dollfus, 1894)

\section{Cymodoce robusta Nierstrasz, 1918}

Diagnosis: Monod (1931: Mem. Soc. Sci. Nat. Maroc, 29, pág.: 54-55, fig.: 50-52)

MATERIAL ESTUDIADO: I(04), 20.II.88: nivel mesolitoral inferior, sobre Chondrus crispus, $1 \mathrm{M}$ de $9,5 \times 4,5 \mathrm{~mm}$. I(05), 20.VIII.88: nivel mesolitoral inferior, $1 \mathrm{M}$ de $8,7 \times 4,5 \mathrm{~mm}$. I(17), 28.VIII.88: nivel mesolitoral inferior, entre talos de Himanthalia elongata $1 \mathrm{M}$ de 8,9x4,5 mm; sobre Chondrus crispus $1 \mathrm{M}$ de $9,4 \times 4,5 \mathrm{~mm}$ y sobre Corallina elongata 1 ejemplar M de 1,0x4,1 mm.

DisCusión: Aunque con ciertas dudas, Torelli (1930) sinonimiza $C$. robusta con $C$. truncata Leach, 1814. Monod (1931) figura tres ejemplares de $C$. robusta provenientes, dos de Marruecos y uno de Cabo Blanco, afirmando que la forma del pleotelson y los urópodos son suficientes para separarla de $C$. truncata, opinión que nosotros compartimos. Además, consideramos que ambas especies se pueden diferenciar por otros caracteres. Los penes son más cortos y anchos en la base en $C$. robusta que en $C$. truncata. El tamaño del apéndice masculino del segundo pleópodo en $C$. robusta sobrepasa claramente la longitud del endopodio, mientras que en $C$. truncata es similar. La ornamentación de tubérculos es fuerte a partir del segundo terguito en $C$. robusta, acompañado de un 
engrosamiento de la parte distal del segmento, mientras que en $C$. truncata es más tenue y aparece sólo en los últimos segmentos del pereion. La forma de la parte anterior del pleon ofrece también claras diferencias entre ambas especies, en C.truncata hay dos netos dientes centrales, ligeramente engrosados con tubérculos, mientras que $C$. robusta presenta cuatro mamelones redondeados; además, el espacio que queda entre los dos centrales es prácticamente recto y muy tuberoso, mientras que en $C$. truncata es claramente cóncavo.

Autoecología: Nierstrasz (1918) y Monod (1931) recogen ejemplares sobre espongiarios y algas (Cystoseira), en localidades submareales a 18 $m$ de profundidad. Sin embargo nuestros ejemplares han sido recogidos en el intermareal inferior en localidades expuestas y semiexpuestas y siempre entre algas (Corallina elongata, Chondrus crispus, Himanthalia elongata y Codium sp.).

DistRIBUCIÓN: Nierstrasz (1918) y Monod (1931) citan esta especie de las islas de Cabo Verde, Cabo Blanco y costas de Marruecos. Nuestros ejemplares constituyen la primera mención en aguas de la Península Ibérica y amplían considerablemente su área de distribución septentrional.

\section{Cymodoce tuberculata Costa, 1851}

Diagnosis: Dumay (1972b, Tethys 4 (2), pág.: 466-469, Pl.: IV-VI).

Material estudiado: D3, 2 juveniles (5,6x3-5,2x2,7 mm), 1 premacho de $6,8 \times 3,3 \mathrm{~mm}$ y $1 \mathrm{M}$ de $8 \times 4 \mathrm{~mm}$. D11, $2 \mathrm{MM}$ de $7,9 \times 4,1 \mathrm{~mm}$.

Autoecología: Recogida en el infralitoral de la boca de la ría, entre 11 y $20 \mathrm{~m}$ de profundidad, en arena de Amphioxus, lo que concuerda con lo observado por Dumay (1971) y Dounas \& Koukouras (1986), que la encuentran en profundidades moderadas.

DisTRIBUCión: Sólo hemos encontrado citas de esta especie en las costas europeas del Mediterráneo (Dumay, 1971 y Dounas \& Koukouras, 1986), representando nuestra cita la primera mención en las costas atlánticas.

\section{Janiropsis breviremis Sars, 1899}

DiAGNOSIS: Holthuis (1956, In: Fauna van Nederland, XVI Isopoda y Tanidacea. Pág.: 110-112, fig.: 37 y 38 Ed. A.W. Sijthoff's Uitgevermaatschappij N.V., Leiden).

Material estudiado: I(01), 31.VII.88: en el mesolitoral inferior, entre Corallina elongata, $1 \mathrm{M}$ de 2,7x0,9 mm. I(17), 18.II.88: en el mesolitoral inferior, entre Corallina elongata, 1 M de 3x1 mm. 30.VII.88: entre rizoides de Laminaria sp. 1 premacho de $1,7 \times 0,6 \mathrm{~mm}$ y 9 ejemplares $\mathrm{MM}(2,9 \times 0,9-1,3 \times 0,4$ $\mathrm{mm})$.

Discusión: Los ejemplares estudiados no presentaban el primer par de pereiópodos diferenciado, ni un alargamiento en los últimos segmentos del pedúnculo antenal descrito por Sars (1899), Naylor (1972) y Kussakin (1988), sin embargo, la determinación específica, basándonos en la foma del primer y segundo pleópodo, forma general del cuerpo, longitud de los urópodos, entre otros caracteres, nos lleva a considerar con certidumbre a nuestros ejemplares como pertenecientes a este taxón, opinando que la ausencia de los caracteres anteriormente mencionados puede ser debida a la diferencia de talla de nuestros ejemplares $(3 \mathrm{~mm})$ frente a la dada por los autores citados $(6 \mathrm{~mm})$.

Autoecología: Nuestros ejemplares han sido recolectados en las dos localidades más expuestas de la ría, ubicándose en el mesolitoral inferior y primeros metros del infralitoral entre Corallina elongata y rizoides de Laminaria ochroleuca, hábitat que coincide con el señalado por otros autores (Sars, 1899; Naylor, 1972). Wolff (1962) la recoge en dragados entre 23 y $125 \mathrm{~m}$ de profundidad.

DistRIBUCIÓN: Se extiende por el Atlántico europeo, desde Noruega hasta el canal de La Mancha, incluyendo las costas británicas e irlandesas. Con la captura del esta especie en la Ría de Ferrol, además de mencionarla por primera vez para aguas ibéricas, ampliamos su límite de distribución meridional.

\section{Paramunna bilobata Sars, 1866}

Diagnosis: Sars (1899: An account of the Crustacea of Norway II. Isopoda. Pág.: 12, Pl.: 47. In: Bergen ed, Publ. Bergen Mus.).

Material estudiado: D19, 1 juvenil de $0,8 \mathrm{x} 0,4 \mathrm{~mm}, 5$ MM $(1,1 \times 0,5-1 \times 0,5 \mathrm{~mm})$ y $5 \mathrm{HH}(1,8 \times 0,8-1,4 \times 0,4 \mathrm{~mm})$. D25, 3 juveniles $(0,8 \times 0,5-0,8 \times 0,4 \mathrm{~mm})$ y $1 \mathrm{M}$ de $1,0 \times 0,5 \mathrm{~mm}$.

Autoecología: Especie infralitoral, recolectada en la parte interna del canal de la ría en fondos de 
arena fangosa y grava, con altos contenidos en carbonato cálcico, entre 17 y $21 \mathrm{~m}$ de profundidad. Sars (1899) la define como una especie frecuente en profundidades moderadas, aunque se la puede encontrar hasta los $200 \mathrm{~m}$ de profundidad, lo que coincide con lo observado por Wolff (1962).

DistRIBUCIÓN: Las escasas referencias bibliográficas de esta especie parecen limitarse a la costa atlántica europea, desde Noruega al Canal de La Mancha, incluyendo las costas inglesas e irlandesas. Nuestra cita es la primera para la Península Ibérica y la localidad más meridional mencionada hasta el momento.

\section{Janira alta (Stimpson, 1853)}

Diagnosis: Carvacho (1981: Crustaceana 41 (2), pág.134, 140-141, fig.: 1b).

Material estudiado: D17, $4 \mathrm{HH}(2,8 \mathrm{x} 0,8-2,2 \mathrm{x} 0,5 \mathrm{~mm}) \mathrm{y}$ $1 \mathrm{M}$ de 1,5x0,4 MM. D19, $2 \mathrm{HH}(2,7 \times 0,8-1,9 \times 0,6 \mathrm{~mm})$. D25, $2 \mathrm{HH}(1,7 \times 0,5-1,4 \times 0,4 \mathrm{~mm})$.

Autoecología: Se localiza en los fondos del canal de la ría, en sustratos de sedimentos gruesos con alto contenido en carbonato cálcico, entre 17 y $25 \mathrm{~m}$ de profundidad. Wolff (1962) indica que esta especie puede encontrarse desde 0 a $1.384 \mathrm{~m}$, soportando temperaturas entre 1 y $21^{\circ} \mathrm{C}$.

DisTRIBUCIÓN: Han sido escasas las referencias que hemos encontrado acerca de la distribución de esta especie en la bibliografía consultada; parece estar bien establecida en las costas del Atlántico de América del Norte (Wolff, 1962; Carvacho, 1981) y en aguas frías del Atlántico oriental: oeste de Islandia (Wolff, opus cit.) y oeste de Irlanda (Carvacho, opus cit.). Nuestra cita contituye la primera mención para las costas ibéricas, a la vez que ampliamos su límite meridional de distribución.

\section{Eurydice affinis (Hansen, 1905)}

Diagnosis: Salvat (1966: Actes Soc. Linn. Bordeaux, 103 A (1), 77 pp.).

Material estudiado: I(03), 2.VIII.88: en sustrato arenoso se ha recogido 1 premacho de $3,8 \times 1,6 \mathrm{~mm}$. I(04), 15.V.88: en sustrato arenoso, $13 \mathrm{MM}(5 \times 1,7-3,8 \times 1,5 \mathrm{~mm}), 1 \mathrm{H}$ de $4,9 \times 2$ $\mathrm{mm}$ y 8 adultos indiferenciados $(4 \times 1,6-2,7 \times 1,1 \mathrm{~mm})$. I(05), 30.VII.88: en un sustrato arenoso $1 \mathrm{M}$ de $4,7 \times 1,7 \mathrm{~mm}$ y $2 \mathrm{HH}$ $(5,3 \times 2,1-5,2 \times 2,1 \mathrm{~mm})$.
Autoecología: Nuestros ejemplares han sido capturados en la playa de Cariño, con un sedimento formado por arena gruesa (10-20\%), arena media $(30-50 \%)$ y arena fina $(25-55 \%)$ en la pequeña acumulacion arenosa de Fornelos, y en la playa de Laxe, con un sedimento formado por arena muy gruesa $(5-10 \%)$, arena gruesa $(5-10 \%)$, arena media (30-50\%) y arena fina (20-25\%); todas ellas estaciones situadas en la margen norte de la ría, con salinidades oceánicas y aguas limpias bien oxigenadas y expuestas a los vientos predominantes del suroeste, condiciones coincidentes con las señaladas por Peres \& Picard (1964); Holdich (1981); Wolff $(1966,1973)$ y Jones \& Naylor (1967).

DISTRIBUCIÓN: Eurydice affinis se distribuye por las costas atlánticas, desde el mar de Norte al Mediterráneo occidental y norte de Africa. La única cita próxima a nuestras costas es la de Monod (1922) en el golfo de Vizcaya, sin precisar localidad, siendo por lo tanto la nuestra la primera mención fehaciente para aguas ibéricas.

\section{Ischyromene lacazei, Racovitza, 1908}

Diagnosis: Racovitza (1908: Arch. Zool. exp. gèn. Paris (4) 9 (Notes et Revue) LX-LXIV). Harrison \& Holdich (1982: Jour. Crust. Biol. 2 (1), pág.: 87-89, fig.: 1(o-s)).

MATERIAL ESTUDIADO: I(03), 2.VIII.88: sobre L. ochroleuca $1 \mathrm{M}$ de 4,6x2,1 mm. I(04), 29.VIII. 88 : 2 juveniles $(2,8 \times 1,5-$ $2,4 \times 1,3 \mathrm{~mm})$ y $2 \mathrm{HH}(4,6 \times 2,3-4,3 \times 2 \mathrm{~mm})$. I(12), 1.VII.88: 3 HH $(4,6 \times 2,1-4,5 \times 2 \mathrm{~mm})$ y $1 \mathrm{M}$ de $4,9 \times 2,3 \mathrm{~mm}$. 1.VIII.88: 1 juvenil de 3,9x1,8 mm. I(15), 20.I.88: sobre L. ochroleuca 2 juveniles $(3,1 \times 1,4-2,6 \times 1,2 \mathrm{~mm}), 7 \mathrm{HH}(4,7 \times 2-3,5 \times 1,7 \mathrm{~mm}), 4$ premachos $(4,8 \times 2,1-4,4 \times 2,1 \mathrm{~mm})$ y $1 \mathrm{M}$ de $5,2 \times 2,5 \mathrm{~mm}$. I(17), 18.II.86: sobre Corallina elongata 35 juveniles $(3,5 \times 1,5-$ $1,3 \times 0,6 \mathrm{~mm}), 2 \mathrm{HH}(4,6 \times 2-4,5 \times 2 \mathrm{~mm}), 9$ premachos $(5,7 \times 2,5-$ $5 \times 2,2 \mathrm{~mm})$ y $7 \mathrm{MM}(5,7 \times 2,5-5 \times 2,3 \mathrm{~mm}) .21 . X I .87$ : en L. pinnatifida, 3 juveniles $(2,7 \times 1,2-1,5 \times 0,7 \mathrm{~mm}), 1 \mathrm{H}$ de $4,5 \times 2,3 \mathrm{~mm}$ y 1 premacho de 3,5x1,3 mm. 18.II.88: en Mytilus edulis 12 juveniles $(3,6 \times 1,6-1,7 \times 0,7 \mathrm{~mm}), 3 \mathrm{HH}(4,5 \times 2-4,3 \times 2 \mathrm{~mm}), 5$ premachos $(5,1 \times 2,5-3,7 \times 1,8 \mathrm{~mm})$ y $2 \mathrm{~mm}(5,6 \times 2,7-4,9 \times 2,3$ mm). 16.V.88: sobre mejillón 4 juveniles $(2,9 \times 1,2-1,8 \times 0,7$ $\mathrm{mm}), 4 \mathrm{HH}(4 \mathrm{x} 1,9-3,8 \times 1,4 \mathrm{~mm}), 4$ premachos $(4,4 \times 2-3,5 \times 1,7$ $\mathrm{mm})$ y $5 \mathrm{MM}(5,9 \times 2,7-4,5 \times 2 \mathrm{~mm})$; sobre L. pinnatifida 16 juveniles $(3,3 \times 1,4-1,6 \times 0,8 \mathrm{~mm}), 16 \mathrm{HH}(4,8 \times 2,4-3,6 \times 1,6 \mathrm{~mm})$ y $6 \mathrm{MM}(6 \times 2,8-4,7 \times 2,2 \mathrm{~mm})$; entre $C$. tomentosum $1 \mathrm{M}$ de $5,3 \times 2,4 \mathrm{~mm}$; sobre $F$. vesiculosus, 9 juveniles $(3,8 \times 1,8-2 \times 0,8$ $\mathrm{mm}), 10 \mathrm{HH}(5 \times 2,2-4,2 \times 2,1 \mathrm{~mm}), 2$ premachos $(4,8 \times 2,1-$ $4,5 \times 2,1 \mathrm{~mm})$ y $3 \mathrm{~mm}(5,7 \times 2,7-4,6 \times 2,1 \mathrm{~mm}) .30$.VII.88: en $M$. edulis $8 \mathrm{HH}(4,5 \times 2-4 \times 1,7 \mathrm{~mm}), 2$ premachos $(5 \times 2,3-3,3 \times 1,4$ mm) $3 \mathrm{MM}(5,9 \times 2,8-5 \times 2,1 \mathrm{~mm})$; sobre $C$. elongata 75 juveniles $(3,2 \times 1,5-1,3 \times 0,6 \mathrm{~mm}), 58 \mathrm{HH}(4,7 \times 2,1-4 \times 2 \mathrm{~mm}), 10$ premachos $(4,7 \times 2,2-3,7 \times 1,6 \mathrm{~mm})$ y $13 \mathrm{MM}(6,3 \times 2,8-4,8 \times 2,2$ $\mathrm{mm})$; en L. ochroleuca 24 juveniles (3,1x1,5-1,4x0,6 mm), 6 
HH ( $5 \times 2,3-4,1 \times 1,9 \mathrm{~mm}), 6$ premachos $(4,7 \times 2,2-3,4 \times 1,6 \mathrm{~mm})$ y $3 \mathrm{MM}(5,3 \times 2,4-5,1 \times 2,3 \mathrm{~mm})$. 28.VIII.88: en una pared extraplomada $5 \mathrm{HH}(4,8 \times 2,3-4 \times 1,8 \mathrm{~mm}), 2$ premachos $(5 \times 2,3-$ $4,9 \times 2,2 \mathrm{~mm})$ y $11 \mathrm{MM}(5,7 \times 2,8-4,3 \times 2,1 \mathrm{~mm})$; en M. edulis 10 juveniles $(3 \times 1,8-1,6 \times 0,7 \mathrm{~mm}), 4 \mathrm{HH}(4,3 \times 2,1-3,9 \times 1,8 \mathrm{~mm})$ y 4 MM (5,9x2,8-5x2,2 mm). 23.XI.88, M. edulis 20 juveniles $(2,5 \times 1,1-1,3 \times 0,6 \mathrm{~mm}), 16 \mathrm{HH}(4,7 \times 2-3,8 \times 1,8 \mathrm{~mm}), 1$ premacho de $4 \times 1,8 \mathrm{~mm}$ y $3 \mathrm{MM}(5,1 \times 2,4-4,1 \times 2,1 \mathrm{~mm})$; sobre $C$. tomentosum 17 juveniles $(3,1 \times 1,5-1,6 \times 0,6 \mathrm{~mm}), 1$ premacho de $3,9 \times 1,8 \mathrm{~mm}$ y $4 \mathrm{MM}(6,2 \times 1,9-4,7 \times 2,1 \mathrm{~mm})$. 30.XI.90: en $C$. elongata 131 juveniles $(3,1 \times 1,3-1,4 \times 0,6 \mathrm{~mm}), 33 \mathrm{HH}(5,2 \times 2,5-$ $3,5 \times 1,6 \mathrm{~mm}), 17$ premachos $(4,5 \times 1,9-3,4 \times 1,5 \mathrm{~mm})$ y $16 \mathrm{MM}$ $(5,2 \times 2,6-4,2 \times 2 \mathrm{~mm})$; en $M$. edulis 33 juveniles $(3,3 \times 1,3-$ $1,3 \times 0,6 \mathrm{~mm}), 1 \mathrm{H}$ de $3,7 \times 1,8 \mathrm{~mm}$ y 1 premacho de $3,7 \times 1,5$ $\mathrm{mm}$.

Autoecología: Peres \& Picard (1964) encuentran esta especie, en el Mediterráneo, en el nivel mediolitoral inferior, entre Mitylus galloprovincialis, mientras que Lombas \& Anadón (1985) y Arrontes \& Anadón (1990a y b) indican su predominancia en los niveles supralitoral y mesolitoral superior. En la Ría de Ferrol esta especie aparece desde el nivel mesolitoral superior hasta los primeros metros del infralitoral entre rizoides de Laminariáceas y claramente asociada a condiciones expuestas o semiexpuestas. Fuera de este hábitat expuesto la hemos encontrado en Mugardos, localidad caracterizada como protegida, entre Fucus vesiculosus.

Distribución: Conocida del Mediterráneo (Racovitza, 1908; Peres \& Picard, 1964). En la Península Ibérica fue citada en Asturias por Lombas \& Anadón (1985) y por Arrontes \& Anadón (1990a y b). Se trata de la primera cita de esta especie en las costas de Galicia.

\section{Cymodoce emarginata sensu Torelli, 1928}

Diagnosis: Torelli (1928a: Boll. Soc. Nat. Napoli 40 (42), pág.: 61). Dumay (1972a: Tethys (4) 1, pág.:133-137, pl.: IIIIV).

Material ESTUdiado: I(04), 29.VIII.88: en un sustrato arenoso colonizado por Ceramiun sp., 11 juveniles $(7 \times 3,5-4,2 \times 2,4$ mm, 2 premachos $(8,2 \times 4,3-7,6 \times 3,8 \mathrm{~mm})$ y $1 \mathrm{M}$ de $10,3 \times 4,7$ mm. I(17), 21.X.87: sobre Pterosiphonia sp. $1 \mathrm{M}$ de 10x4,2 $\mathrm{mm}$; en una pared extraplomada del mesolitoral inferior 1 juvenil de 9,5x4,6 mm. 28.VIII.88, en un extraplomo del horizonte mesolitoral medio 112 juveniles $(9 \times 4,2-4,4 \times 2,5 \mathrm{~mm}), 2$ premachos $(9 \times 4,5-8,4 \times 4,4 \mathrm{~mm})$ y $4 \mathrm{MM}(11,3 \times 4,6-9,6 \times 4 \mathrm{~mm})$; sobre Chondrus crispus $1 \mathrm{M}$ de 10,3x4,6 mm. 23.IX.88: sobre Laurencia pinnatifida 3 juveniles $(9,5 \times 4,4-6,3 \times 3,2 \mathrm{~mm})$ y $1 \mathrm{M}$ de $10,6 \times 4,4 \mathrm{~mm}$; en una pared extraplomada del mesolitoral inferior 1 juvenil de 9,4x4 mm y $3 \mathrm{MM}(10,8 \times 4,7-9,7 \times 4,5$ $\mathrm{mm})$.
Discusión: Dumay (1971) opina que los ejemplares identificados por Torelli (1930), como $C$. emarginata (Leach 1818), se corresponden a una nueva especie, perfectamente distingible de $C$. truncata por la forma y longitud del estilete copulador del segundo pleópodo, forma de los urópodos, tridentura pleotelsónica y ornamentación del pleon. Nosotros coincidiendo con la opinión de Dumay (opus cit.), y siguiendo su criterio, la denominamos como $C$. emarginata sensu Torelli, apuntando que esta cuestión deberá ser resuelta en un futuro próximo con el estudio de los paratipos.

Autoecología: Nuestras observaciones son coincidentes con las hechas por Dumay (1971) sobre esta especie, que hemos recogido en el mesolitoral inferior de dos estaciones caracterizadas como semiexpuesta y expuesta, apareciendo protegida de la luz en los extraplomos o entre algas.

DisTRIBUCIÓN: De la bibliografía consultada se desprende que esta especie está ampliamente distribuida por todo el Mediterrámeo y a lo largo de las costas francesas y británicas. En la Península Ibérica Castelló (1986b) la cita en las costas de Cataluña y en el archipiélago balear (isla de Ibiza). Su hallazgo en la Ría de Ferrol supone la primera mención de esta especie en aguas del Atlántico ibérico.

\section{Cymodoce tattersalli Torelli, 1928}

Diagnosis: Torelli (1928: Boll. Soc. Nat. Napoli 40 (2), pág.: 62). Dumay (1972b: Tethys 4 (2), pág.: 458-462, 469470, pl.: I,II).

Material estudiado: D1, 4 ejemplares juveniles (5,7x2,9$3,4 \times 1,6 \mathrm{~mm})$. D15, 8 adultos indiferenciados $(5,9 \times 2,8-2,8 \times 1,2$ $\mathrm{mm})$. D17, 25. VII.87, en el infralitoral a $25 \mathrm{~m}$ de profundidad: 21 adultos indiferenciados $(5,2 \times 2,7-3,8 \times 2,2 \mathrm{~mm}), 2$ premachos $(6 \times 3-4,9 \times 2,5 \mathrm{~mm})$ y $12 \mathrm{MM}(7,5 \times 3,5-6,1 \times 3 \mathrm{~mm}) .12 . V \cdot 93: 11$ adultos indiferenciados $(4,5 \times 2,2-5,3 \times 2,6 \mathrm{~mm})$ y $1 \mathrm{M}$ de $8,4 \times 3,6 \mathrm{~mm}$.

Autoecología: Dounas \& Koukouras (1986) la recogen en pequeñas depresiones superficiales de las esponjas, mientras que nuestros ejemplares proceden de fondos con un alto contenido en carbonato cálcico y un tipo sedimentario medio o grueso, a profundidades comprendidas entre 7 y $25 \mathrm{~m}$, en una zona del canal sometida a fuertes corrientes mareales; no obstante en la estación D1 la recolectamos en un fondo de arena fina con conchas. 
DisTRIBUCión: Mencionada en las costas inglesas por Tattersal (1905) y en el Mediterráneo por Dumay (1971; 1972b), Torelli (1930), Bacesco (1958), Dounas \& Koukouras (1986) y Castelló (1986b). Esta es su primera cita en aguas del Atlántico ibérico.

\section{Jaera nordica nordica Lemercier, 1960}

DiAgnosis: Renault (1958: Arch. Zool. exp. gèn., 96: 6370). Lemercier (1960: Crustaceana 1: 19-22, fig: 3b, 4 A2 y B2, 5-7.

Material estudiado: I(09), 14.V.88: en el intermareal, sobre Fucus ceranoides 6 juveniles $(1,95 \times 0,8-1 \times 0,4 \mathrm{~mm}), 21$ $\mathrm{HH}(3,9 \times 1,75-1,85 \times 0,8 \mathrm{~mm})$ y $4 \mathrm{MM}(3,35 \times 1,8-3,1,65 \mathrm{~mm})$; en el infralitoral, también sobre $F$. ceranoides 8 premachos $(2,23 \times 1,05-2,35 \times 1,2 \mathrm{~mm}), 10 \mathrm{MM}(3,25 \times 1,8-2,95 \times 1,6 \mathrm{~mm})$ y $8 \mathrm{HH}(3,35 \times 1,05-2,05 \times 1,05$ mm). 25.IX.88: en Fucus ceranoides infralitoral 6 juveniles $(1,8 \times 0,75-1,55 \times 0,55 \mathrm{~mm}), 26 \mathrm{MM}$ $(3,7 \times 2,1-3,4 \times 1,9 \mathrm{~mm})$ y $48 \mathrm{HH}(3,8 \times 1,95-1,75 \times 0,8 \mathrm{~mm})$. I(16), 15.V.88: bajo piedras en una charca con aporte de agua dulce, 8 juveniles $(1,7 \times 0,75-1,5 \times 0,6 \mathrm{~mm}), 6 \mathrm{MM}(3,5 \times 2,05-$ $3,3 \times 1,9 \mathrm{~mm})$ y $5 \mathrm{HH}(3,45 \times 1,75-2,25 \times 1,05 \mathrm{~mm})$.

Autoecología: Los trabajos acerca de los requerimientos del hábitat de esta especie no son abundantes. Renault (1958) y Lemercier (1960) coinciden al observar, que ocupa los niveles más altos del horizonte intermareal (por encima de las especies del "grupo albifrons") y la localizan principalmente en localidades de aguas someras estuáricas.

Nuestros datos son coincidentes en cuanto a que también la hemos encontrado en aguas someras de baja salinidad, pero no con respecto al nivel intermareal que ocupan, ya que en la estación de Xubia (I09), sobre Fucus ceranoides, tanto intermareal como infralitoral, ha sido donde más abundantemente apareció, siendo sin embargo muy poco frecuente bajo piedras y rocas. No hemos observado competencia con J. albifrons ya que no aparece en esta estación. Hemos recogido esta especie en la estación Cabaliños (I16) en condiciones totalmente diferentes, en la que el agua dulce proviene de la escorrentía de la ladera de Montefaro, formando pequeñas charcas de agua dulce en la zona supralitoral, en las que se encuentra bajo piedras. Destacamos que en el mismo nivel y en pozas salinas con similares características habita J. albifrons.
Por ello, creemos que la única exigencia clara de esta especie es una baja salinidad, ocupando niveles intermareales y substratos diferentes dependiendo de la localidad.

DiSTRIBUCIÓN: Existen pocos datos sobre la repartición de esta especie. En general parece confinada a puntos dispersos de las costas francesas, danesas e inglesas (véase Bate \& Westwood, 1866-68; Arcangeli, 1934; Renault, 1958 y Lemercier, 1960).

El único dato sobre su distribución en el litoral ibérico se debe a Veuille (1979) que la señala en el País Vasco, sin precisar localidad, por lo cual, con nuestras capturas la citamos por segunda vez para Atlántico ibérico al tiempo que ampliamos su límite de distribución meridional.

\section{Jaera hopeana Costa, 1853}

Diagnosis: Veuille (1979, Bijd. Dierk., 49 (2), pág.: 198202, fig.: 2d, 2f; 3 y 4a).

MATERIAL ESTUDIADO: I(04), 25.V.87: sobre $F$. vesiculosus $1 \mathrm{H}$ de $1,05 \times 0,45 \mathrm{~mm}$ y $1 \mathrm{M}$ de $1 \times 0,45 \mathrm{~mm}$. I(05), 22.I.88: sobre Sphaeroma serratum, $5 \mathrm{HH}(0,95 \mathrm{x} 0,45-0,94 \mathrm{x} 0,4 \mathrm{~mm})$ y $3 \mathrm{MM}(0,95 \times 0,4-0,95 \times 0,35 \mathrm{~mm})$. I(11), 23.VII.87: F. vesiculosus, $15 \mathrm{HH}(1 \times 0,5-1 \times 0,45 \mathrm{~mm}), 8 \mathrm{MM}(1,5 \times 0,6-1,35 \times 0,55$ $\mathrm{mm})$. I(12), 2.VII.88: sobre $S$. serratum, $1 \mathrm{H}$ de $1 \times 0,5 \mathrm{~mm}$. I(14), 22.VII.87: bajo piedras, $1 \mathrm{M}$ de 1,65x0,6 mm.

Autoecología: Jaera hopeana vive característicamente asociada a Sphaeroma serratum, que le ofrece abrigo y medio de transporte, lo que conduce a considerar esta asociación como un caso de foresia. Por otro lado, tanto nuestros datos como los de los autores consultados (Prunus \& Pantoustier, 1977; Veuille, 1979; Hahtela \& Naylor, 1965; Geldiay \& Kocatas, 1972), coinciden al poner de manifiesto el carácter no obligatorio de esta asociación, ya que encontramos tanto a $S$. serratum desprovista de $J$. hopeana en cualquier biotopo y estación, como a la especie J. hopeana protegida entre algas y biso de mejillón.

También concuerdan nuestras observaciones con las de Prunus \& Pantoustier (1977) al precisar que esta especie se encuentra en la cara ventral de Sphaeroma, variando su posición durante los desplazamientos del huésped; así J. hopeana se fija en zonas bien protegidas, entre los pleópodos, cuando Sphaeroma camina y, entre los pereiópodos, cuan- 
do éste nada, mientras que ocupa espacios más amplios en la cara ventral cuando el huesped está enrollado. Son frecuentes, por tanto, los desplazamientos de J. hopeana por el cuerpo de $S$. serratum, incluso por la cara dorsal del mismo (Prunus \& Pantoustier, opus cit.)

En cuanto a los requerimientos ecológicos, Valkanov (1938) la señala como una especie muy eurióica fijando un rango de salinidad entre 8 y $35 \%$. Haathela \& Naylor (1965) señalan la necesidad de un aporte de agua dulce, aunque éste sea esporádico, en las estaciones donde está presente la especie. En la Ría de Ferrol parece comportarse de forma ligeramente diferente, ya que no aparece en las localidades más internas y fangosas de la ría, a pesar de la presencia de S. serratum. La hemos recolectado en estaciones semibatidas o semiprotegidas, con una preferencia por substratos arenosos $\mathrm{o}$ areno-fangosos y aguas limpias de media y alta salinidad.

DistribuCIÓN: Prunus \& Pantoustier (1976) citan esta especie en puntos muy diversos de las costas mediterráneas, mar Negro y costas francesas de La Mancha. Hahtela \& Naylor (1965) la mencionan en la costa atlántica de Marruecos, canal de La Mancha y costas inglesas. Veuille \& Kocatas (1978-1979) lo hacen en la costa de Turquía.

Los únicos datos sobre la geonemia de esta especie en el litoral ibérico son los dados por Castelló (1985), que la menciona por primera vez en Cataluña y posteriormente (Castelló, 1986b) la cita en Formentera. La ausencia de otras menciones nos inclinan a considerarla como primera cita para el litoral atlántico ibérico.

\section{Referencias}

ArCANGELI, A., 1934. Il genere Jaera Leach nel Mediterraneo e la convivenzia occasionale di Jaera hopeana Costa con Sphaeroma serratum (Fabr.) (Isopodi marini). Boll. Mus. Zool. Anat. Comp. Torino, 44: 273-292.

Arrontes, J. \& ANADÓN, R., 1990a. Seasonal variation and population dynamics os Isopods inhabiting intertidal macroalgae. Sci. Mar., 54(3): 231-240.
Arrontes, J. \& ANADÓN, R., 1990b. Distribution of intertidal isopods in relation to geographical changes in macroalgal cover in the bay of Biscay. J. Mar. Biol. Ass. U.K., 70: 283-293.

BACESCO, M., 1958. Les rèpresentants du genre Cymodoce (Crustacea; Isopoda) de la mer Noire. Vie Milieu, 1958: 431-440.

Bate C.S. \& Westwood, J.O., 1866-1868. II. Isopoda 1868 (Tanaidae, Anthirudae, Ancenidae, 1866 Bopyridae Aegidae, Asellidae, Arcturidae, Idoteidae 1867 Sphaeromidae, Oniscidae, 1868: 99-495. In: J. Van Worst Paternoster Rorv. (eds). British sessile-eyed Crustacea, London, pp: 99-494

Carvacho, A., 1981. Le genre Janira Leach avec description d'une nouvelle espèce (Isopoda, Asellota). Crustaceana, 41(2): 131-142.

Castelló, J., 1984. Sobre la fauna de Crustáceos Isópodos litorales de Cataluña y Baleares (I): Valvifera; Oniscoidea. Publ. Dept. Zool. Barcelona, 10: 27-37.

Castelló, J., 1985. Sobre la fauna de Crustáceos Isópodos litorales de Cataluña y Baleares (II): Asellota; Anthuroidea. Publ. Dept. Zool. Barcelona, 11: 29-35.

Castelló, J., 1986a. Sobre la fauna de Crustáceos Isópodos litorales de Cataluña y Baleares (III): Flabellifera. Publ. Dept. Zool. Barcelona, 12: 59-59.

CAstelló, J., 1986b. Contribución al conocimiento biológico de los Crustáceos del litoral catalano-balear. Tesis Doctoral. Universidad de Barcelona, España.

Dounas, C. \& Koukouras, A.T.H., 1986. The genus Cymodoce Leach (Isopoda, Flabellifera) in the north Aegean sea. Crustaceana, 30(1): 68-72.

DumaY, D., 1971. Ecologie et biologie du genre Cymodoce (Isopoda; Flabellifera) dans la region de Marseille. Téthys, 2(4): 827-858.

DUMAY, D., 1972a. Révision systématique du genre Cymodoce (Isopoda Flabellifera) 3. Description de $C$. spinosa Risso et de $C$. emarginata sensu Torelli. Téthys, 4(1): 127-144.

DumaY, D., 1972b. Révision systematique du genre Cymodoce (Isopoda Flabellifera) 4. Description 
de C. tattersalli Torelli, C. rubropunctata (Grube), C.tuberculata Costa. Clef systematique et conclusion générale. Téthys, 4(2): 457-480.

Franch, L.A. \& Ballesteros, M., 1993. Aportación al conocimiento de los Isópodos (Crustacea; Isopoda) litorales del Estany des Peix (Formentera). Publ. Esp. Inst. Esp. Oceanogr., 11: 153-157.

GELIDAY, R. \& KoKATAS, A., 1972. Isopods collected in Izmir bay, Aegean sea. Crustaceana, 3: 19-30.

HaAhtela, I. \& NAYloR, E., 1965. Jaera hopeana an interdital Isopod new to the british fauna. $J$. Mar. Biol. Ass. U.K., 45: 367-371.

HARRISON, K. \& HoLDICH, D.M., 1982. Revision of the genera Dynamenella, Ischyromene, Dymanenopsis and Cymodocella (Crustacea; Isopoda); including a new genus and five new species of eubranchiate sphaeromids from Queensland waters.J. Crust.Biol., 2(1): 84-119.

HoLDICH, D.M., 1981. Opportunistic feeding behaviour in a predatory Isopod. Crustaceana, 41(1): 101-103.

HolTHuIs, L.B., 1956.xVI Isopoda y Tanidacea. In: A.W. Sijthoff's Uitgenermaatschappij N.V. (Eds). Fauna van Nederland. Leiden, 279 pp.

IBORRA, B. \& Ros, J., 1984. Primeros datos sobre las poblaciones de Sphaeroma del Mar Menor (Murcia). Actas IV Simp. Ibér. Estud. Bentos Mar., 3: 227-234.

Jones, M.B. \& NAYlor, E., 1967. The distribution of Eurydice (Crustacea, Isopoda) in British waters including $E$. affinis new to Britain. $J$. Mar. Ass. U.K., 47: 373-382.

Junoy, J. \& VieiteZ, J.M., 1988. Crustáceos intermareales de sustrato blando de la ría de Foz (Lugo). Actas III Simp. Ibér. Entomol.: 529540 .

JunOY, J. \& VIEITEZ, J.M., 1990. Idotea chelipes (Pallas, 1866) (Crustacea, Isopoda) en la Ría de Foz (Lugo, España). Bol. R. Soc. Esp. Hist. Nat., (Sec. Biol.), 85(1-4): 87-97.

KusSAKIN, O.G., 1988. Marine and brackish water likefooted Crustacea (Isopoda) from the cold and temperate waters of the nortern hemisphere 3. In: Opredeliteli po fauna SSSR izdervaemye
Zoologischeski Inst. Acad. Nauk. SSSR 152 (Izdatel'stwo Nauka, Leningrad).

LEMERCIER, A., 1960. La super-espèce Jaera nordmanni (Rathke) (Isopodes, Asellotes, Janiridae). Crustaceana, 1: 9-27.

Lombas, I. \& AnAdón, N., 1985. Estudio de la fauna de microhábitats esciáfilos intermareales en Luanco (Asturias). Rev. Biol. Oviedo, 3: $107-$ 120 .

Monod, Th., 1922. Contribution à l'étude faunistique des isopodes de France. Bull. Mus. Natl. Hist. Nat., Paris, 28: 270-362.

MonoD, Th., 1931. Tanaidacés et Isopodes aquatiques de $1^{\prime}$ Afrique occidentale et septentrional. 3 (1) Sphaeromatidae.Mém.Soc.Sci.Nat.Maroc, 29: 7-21, fig: 1-74.

NAYLOR, E., 1972. British marine Isopods. Key and notes for the identification of the speces. In: Synopses of the British Fauna (3). Linnean Society of London \& Academic Press. London.

Nierstrasz, H.F., 1918. Alte und neue Isopoden. Zool. Meded. Rijks Mus. Naturlij. Leiden Del IV, Afl 2: 103-142, pl (IxX ).

Peres, J.M. \& PiCARD, J., 1964. Nouvel manuel de bionomie benthique de la mer Méditerranée. Trav. Est. Marit. Endoume, 31(47): 4-137.

PÉreZ-Cirera, J.L. \& MALdONADO, J.L., 1982. Principales tipos de vegetación y su zonación en el litoral comprendido entre las rías de Camariñas y Corme y Lage (Costa de Camelle, La Coruña). Collectanea Botanica, 13(2): 893910

PÉREZ-Cirera, J.L. \& PACheCO, J., 1985. Zonación y distribución geográfica de la vegetación de la ría de Lires (NW de España). Trab. Compost. Biol. 12: 153-183.

Prunus, G. \& Pantoustier, G., 1976. Le genre Jaera Leach (Isopode, Asellote) sur les côtes de Tunise. Etude morphologique et ecologique. Arch. Zool.exp. gén., 117: 235-254.

Prunus, G. \& Pantoustier, G., 1977. Aspects qualitatifs de l'asociation de deux isopodes littoreaux Jaera hopeana Costa et Sphaeroma serratum Latreille. Bull. Soc. Zool. France, 102(3): 251 . 
RACOVITZA, E.G., 1908. Ischyromene lacazei, n. g., n. sp. Isopoda méditerranéen de la famille des Spheromides. Arch. Zool. exp. gén. Paris (4) 9(Notes et Revue): 60-64.

Renault, O., 1958. Note sur la morphologie et le développement de Jaera nordmanni (Rathke) (Isopode Asellote). Arch. Zool. exp. gén., 96(Notes et Revue): 63-70.

Ros, J., Perez-Ruzafa, A., Marcos, C. \& PerezRuZAFA, I.M., 1987. Resultados preliminares en el estudio del bentos del Mar Menor. Cuad. Marisq. Publ. Téc., 11: 305-321.

Salvat, B., 1966. Eurydice pulchra (Leach, 1915), E. affinis (Hansen, 1905) (Isopodes, Cirolanidae) taxonomie, ethologie, ecologie, repartition vertical et cycle reproducteur. Act. Soc. Linn. Bordeaux , 103(A): 1-77.

SARS, G.O., 1899. II Isopoda. In: Bergen Museum (Eds.). An account of the Crustacea of Norway, with short description and figures of all the species, Bergen, 270 pp, 100 pl.

SEOAne-CambA, J.A., 1969. Sobre la zonación del sistema litoral y su nomenclatura. Inv. Pesq., 33(1): 261-267.

TATTERSALL, W., 1905. The marine fauna of the coast of Ireland. V. Isopoda. Scient. Invest., 2: 53-142. Dept. Agriculture and Technical Instrution for Ireland, Fish. Branch, Dublin.

Torelli, B., 1928-29. Notizie su alcuni Isopodi del golfo di Napoli. Il genere Cymodoce. Bull. Soc. Nat. Napoli, 40(42): 57-64.

Torelli, B., 1930. Notizie su alcuni Isopodi del golfo di Napoli. Il genere Cymodoce. Boll. Soc. Nat. Napoli, 40(42): 57-64.

VAKALANOV, A., 1938. Uebersicht der europaïschen Vertretern der Gattung Jaera Leach 1813 (Isopoda genuina). God. Sof. Univ. Sofiya Fiz. Mat. Fak., 34(3 Sci. nat.): 53-78.

Veuille, M. \& KoKatas, A., 1978-79. Les Jaera (Isopodes, Asellotes) des côtes de Turquie, étude comparative et biogeographique. Vie Milieu, Ser. AB, 28-29(4): 597-611.

Veuille, M., 1979. L'evolution du genre Jaera Leach (Isopodes; Asellotes) et ses rapports avec l'histoire de la Méditerranée. Bijdr. Dierkunde, 49(2): 195-217.
WOLFF, T., 1962. The systematics and biology of bathyal and abyssal Isopoda Asellota. Galathea Report, 6: 1-230.

WolfF, W.J., 1966. Notes on Eurydice (Isopoda, Flabellifera) from the Netherlands. Zool. Med., 41(4): 221-227.

WolfF, W.J., 1973. The stuary as a habitat an analysis of data on the soft-bottom macrofauna of the estuarine area of the rives Rhine, Mense and Scheldt. Zool. Verh., 126: 1-242.

Wommersley, H.B.S. \& Edwards, S.J., 1952. Marine coastal zonation in Southern Australia in relation to a general scheme of classification. $J$. Ecol., 40(1): 84-90.

\section{Apéndice}

A) Número, toponimia y coordenadas sexagesimales de las 17 estaciones intermareales muestreadas:

I(01), Pieiro Pequeno, 4327'19'”N; 08²1'18' W. I(02), Canelas, 4327'49' N; 08 $19^{\circ}$ '57' 'W.

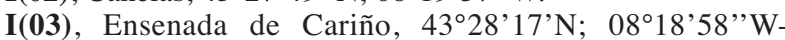
$43^{\circ} 28^{\prime} 17^{\prime \prime} \mathrm{N}$; $08^{\circ} 18^{\prime} 50^{\prime \prime} \mathrm{W}$

I(04), Fornelos, $43^{\circ} 28^{\prime} 09^{\prime \prime} \mathrm{N}$; $08^{\circ} 18^{\prime} 48^{\prime \prime} \mathrm{W}$.

I(05), Ensenada de Laxe, 4327'39'”N; 08¹6'54'”W.

I(06), A Cabana, 4329'36"N; 08¹5'07' 'W.

I(07), A Gándara, 4329'322'’N; 08¹1'38'”W.

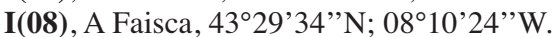

I(09), Xubia, 4330'50'”N; 089'24'”W.

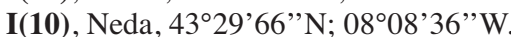

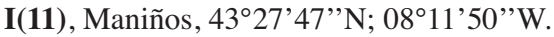

I(12), Mugardos, 43 $27^{\circ} 52^{\prime \prime} \mathrm{N}$; 08 $15^{\circ} 07^{\prime}$ 'W

I(13), A Redonda, 43⒉ $7^{\prime} 52^{\prime} \mathrm{N}$; 08 $06^{\circ} 16^{\prime} 05^{\prime}$ 'W.

I(14), San Martín, 4327'39'”N; 08 $16^{\circ} 57^{\prime \prime} \mathrm{W}$.

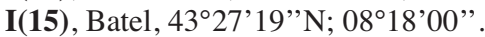

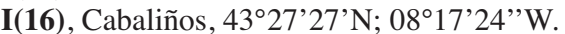

I(17), O Segaño, 4327'22'’N; 08¹9'35'’W.

B) Fecha, coordenadas sexagesimales, profundidad y componentes no texturales del sedimento en las 75 estaciones infralitorales muestreadas:

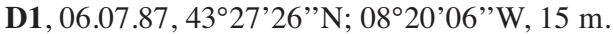
D2, 02.06.87, $43^{\circ} 27^{\prime} 11^{\prime \prime} \mathrm{N}$; 08 $20^{\circ}, 12^{\prime \prime} \mathrm{W}, 34 \mathrm{~m}$.

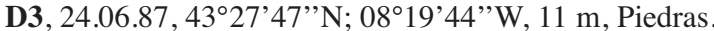

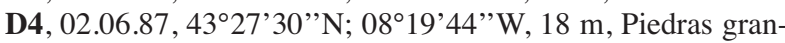
des.

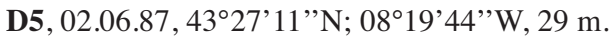
D6, 06.07.87, 4328'06'”N; 08 19'04'’W, 6 m. D7, $11.05 .87,43^{\circ} 27^{\prime} 50^{\prime \prime} \mathrm{N}$; 08 ${ }^{\circ} 19^{\prime} 16^{\prime \prime} \mathrm{W}, 17 \mathrm{~m}$. D8, 06.07.87, 4327'30'”N; 08¹9'16' 'W, 17 m. D9, 02.06.87, 43 $27^{\circ} 11^{\prime \prime} \mathrm{N}$; 08 $19^{\prime} 16^{\prime \prime} \mathrm{W}, 23 \mathrm{~m}$, Piedras. 
D10, $11.05 .87,43^{\circ} 27^{\prime} 50^{\prime \prime} \mathrm{N} ; 08^{\circ} 18^{\prime} 45^{\prime \prime} \mathrm{W}, 18 \mathrm{~m}$.

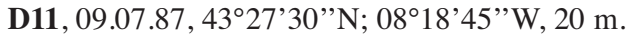

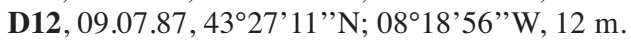

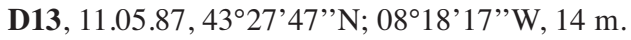

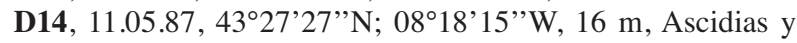
Pecten maximus.

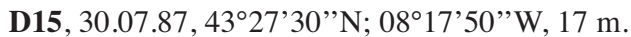

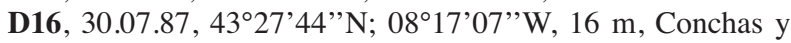
algas.

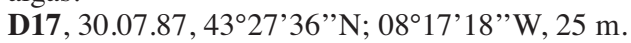

D18， 24.06.87， $43^{\circ} 28^{\prime} 02^{\prime \prime} \mathrm{N} ; \quad 08^{\circ} 16^{\prime} 37^{\prime} \mathrm{W}, \quad 7 \mathrm{~m}$, Branchiostoma lanceolatum.

D19, 05.08.87, 432 $27^{\prime} 49^{\prime \prime} \mathrm{N} ; 08^{\circ} 16^{\prime} 42^{\prime \prime} \mathrm{W}, 21 \mathrm{~m}$.

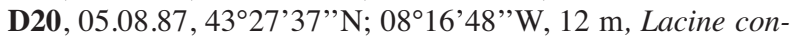
chilega.

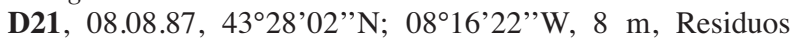
urbanos.

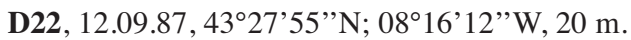

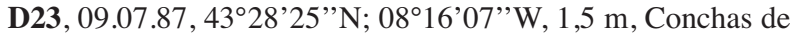
C. edule y algas.

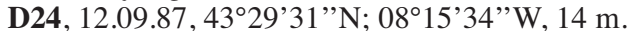
D25, 12.09.87, $43^{\circ} 28^{\prime} 12^{\prime \prime} \mathrm{N}$; $08^{\circ} 15^{\prime} 44^{\prime \prime} \mathrm{W}, 17 \mathrm{~m}$. D25, $12.09 .87,43^{\circ} 27^{\prime} 08^{\prime \prime} \mathrm{N} ; 08^{\circ} 15^{\prime} 37^{\prime \prime} \mathrm{W}, 13 \mathrm{~m}$. D26, $08.08 .87,43^{\circ} 27^{\prime} 52^{\prime \prime} \mathrm{N}$; $08^{\circ} 15^{\prime} 48^{\prime}$ ” $\mathrm{W}, 15 \mathrm{~m}$, L. conchilega. D27, 05.08.87, $43^{\circ} 29^{\prime} 25^{\prime \prime} \mathrm{N}$; $08^{\circ} 15^{\prime} 56^{\prime \prime} \mathrm{W}, 1,5 \mathrm{~m}$.

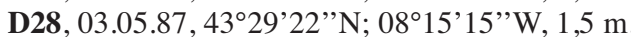
D29, $19.02 .87,43^{\circ} 28^{\prime} 09^{\prime \prime} \mathrm{N}$; $08^{\circ} 15^{\prime} 15^{\prime \prime} \mathrm{W}, 2 \mathrm{~m}$. D30, $19.02 .87,43^{\circ} 28^{\prime} 51^{\prime \prime} \mathrm{N}$; $08^{\circ} 15^{\prime} 15^{\prime \prime} \mathrm{W}, 9 \mathrm{~m}$.

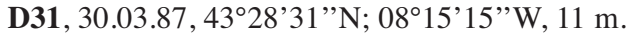
D32, $02.04 .87,43^{\circ} 27^{\prime} 08^{\prime \prime} \mathrm{N}$; $08^{\circ} 15^{\prime} 16^{\prime}$ 'W, $15 \mathrm{~m}$, Turritella comunis.

D33, 01.05.87, $43^{\circ} 29^{\prime} 56^{\prime \prime} \mathrm{N}$; $08^{\circ} 15^{\prime} 06^{\prime}$ 'W, $8 \mathrm{~m}$.

D34, 03.05.87, $43^{\circ} 29^{\prime} 27^{\prime \prime} \mathrm{N}$; 08 $8^{\circ} 14^{\prime} 48^{\prime \prime} \mathrm{W}, 1 \mathrm{~m}$.

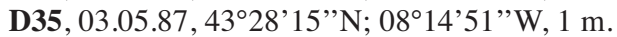

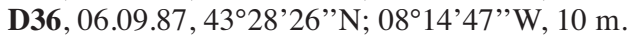

D37, 08.08.87, $43^{\circ} 28^{\prime} 10^{\prime \prime} \mathrm{N}$; 08 $08^{\circ} 14^{\prime} 47^{\prime \prime} \mathrm{W}, 15 \mathrm{~m}$.

D38, $\quad 18.07 .87, \quad 43^{\circ} 27^{\prime} 52^{\prime \prime} \mathrm{N} ; \quad 08^{\circ} 14^{\prime} 47^{\prime \prime} \mathrm{W}, \quad 10 \mathrm{~m}$, Chaetopterus variopedatus.

D39, Ver texto.

D40, Ver texto.

D41, $\quad 06.09 .87, \quad 43^{\circ} 28^{\prime} 10^{\prime \prime} \mathrm{N} ; \quad 08^{\circ} 14^{\prime} 16^{\prime \prime} \mathrm{W}, 13 \mathrm{~m}$, Chaetopleura fulva.

D42, $11.08 .87,43^{\circ} 27^{\prime} 52^{\prime \prime} \mathrm{N}$; 08¹4'16”'W, $13 \mathrm{~m}$, Ch. variopedatus.

D43, Ver texto.

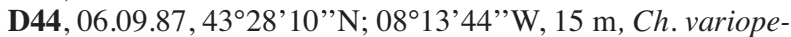
datus, residuos urbanos.

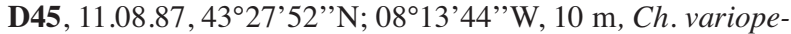
datus y Cerianthus sp.

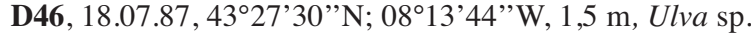

D47, 22.07.87, $43^{\circ} 28^{\prime} 51^{\prime}$ 'N; $08^{\circ} 13^{\prime} 02^{\prime \prime} \mathrm{W}, 1,5 \mathrm{~m}$, Ulva $\mathrm{sp}$.

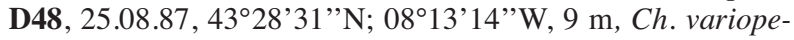
datus.

D49, $11.08 .87,43^{\circ} 28^{\prime} 10^{\prime \prime} \mathrm{N} ; 08^{\circ} 13^{\prime} 14^{\prime \prime} \mathrm{W}, 12 \mathrm{~m}$.

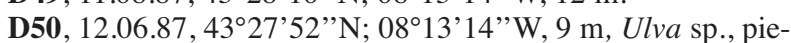
dras y conchas.

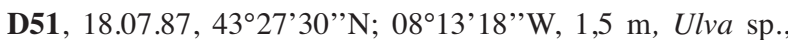
piedras y conchas.

D52, 22.07.87, $43^{\circ} 28^{\prime} 51^{\prime \prime} \mathrm{N}$; $08^{\circ} 12^{\prime} 43^{\prime \prime} \mathrm{W}, 2 \mathrm{~m}$

D53, 22.07.87, $43^{\circ} 28^{\prime} 31^{\prime \prime} \mathrm{N}$; $08^{\circ} 12^{\prime} 43^{\prime \prime} \mathrm{W}, 8 \mathrm{~m}$.

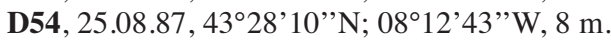

D55, $12.06 .87,43^{\circ} 27^{\prime} 52^{\prime \prime} \mathrm{N}$; $08^{\circ} 12^{\prime} 43^{\prime \prime} \mathrm{W}, 4 \mathrm{~m}$, Conchas.

D56, $03.10 .87,43^{\circ} 27^{\prime} 38^{\prime \prime} \mathrm{N}$; $08^{\circ} 12^{\prime} 43^{\prime \prime} \mathrm{W}, 1 \mathrm{~m}$, Conchas

D57, 25.07.87, $43^{\circ} 28^{\prime} 31^{\prime \prime} \mathrm{N} ; 08^{\circ} 12^{\prime} 14^{\prime \prime} \mathrm{W}, 7 \mathrm{~m}$.

D58, $25.07 .87,43^{\circ} 28^{\prime} 10^{\prime \prime} \mathrm{N}$; $08^{\circ} 12^{\prime} 14^{\prime \prime} \mathrm{W}, 6 \mathrm{~m}$, Ficulina ficus.

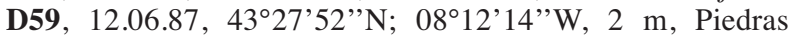
pequeñas.

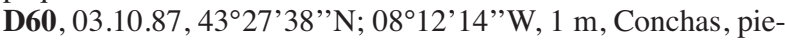
dras, C. edule y P. ulvae.

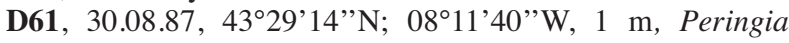
ulvae.

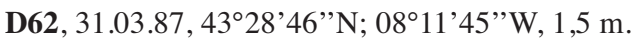

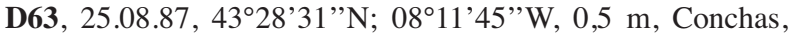
Zostera spp., Ulva sp.

D64, 25.07.87, $43^{\circ} 28^{\prime} 10^{\prime \prime} \mathrm{N}$; 08 ${ }^{\circ} 11^{\prime} 45^{\prime \prime} \mathrm{W}, 2 \mathrm{~m}$, Piedras, conchas y Ulva sp..

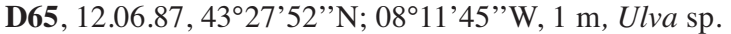

D66, 30.08.87, 43 292'27'N; 08 $11^{\circ} 13^{\prime \prime} \mathrm{W}, 1 \mathrm{~m}$, Conchas.

D67, 31.03.87, 4329'16"N; 08¹1'13”'W, 1,5 m, Zostera spp., piedras y conchas.

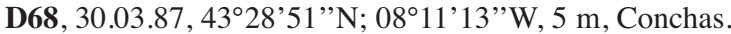

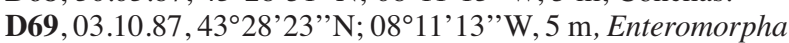
sp.

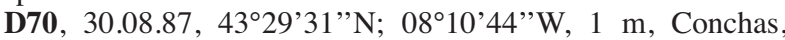
Ulva sp., Zostera spp.

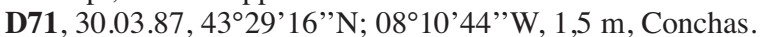

D72, 31.03.87, $43^{\circ} 28^{\prime} 51^{\prime \prime} \mathrm{N}$; 08 $8^{\circ} 10^{\prime} 42^{\prime}$ 'W, 1,5 m, Conchas y piedras.

D73, 30.08.87., 43²9'46”N; 08¹0'15'W, 1,5 m, Piedras, $L$. conchilega, Ulva sp.

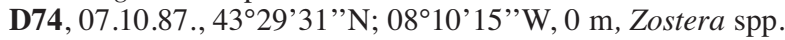

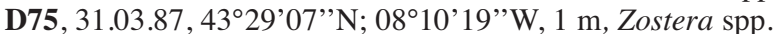

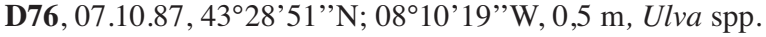

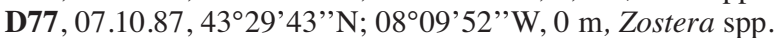

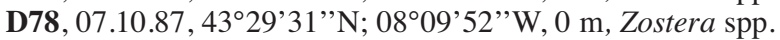

Recibido, el 9-VII-1996 Aceptado, el 19-XI-1996 Publicado, el 27-XII-1996 